\title{
Cognitive Functions in Recipients of Liver Transplantation: Prospective Comparative Study

\author{
${ }^{1}$ Ahmad Saad Mohamed, ${ }^{2}$ Mahmoud Ahmed Elmeteini, ${ }^{1}$ Ghada Abd Elrazek Mohamed, ${ }^{1}$ Doha \\ Mostafa Elserafy, ${ }^{1}$ Reem Elsayed Hashem, ${ }^{1}$ Alaa Adel Elmadani \\ Department of Neuropsychiatry, Faculty of Medicine-Ain Shams University
} \\ ${ }^{2}$ Dean of Faculty of Medicine, Director of Ain Shams Center for Organ Transplant, Professor of Surgery-Ain Shams University Corresponding author: Alaa Adel Elmadani, Mobile: 01005400566; Email: alaa.aelmadani@gmail.com
}

\begin{abstract}
Background: cognitive control refers to the ability to regulate, coordinate, and sequence thoughts/actions in accordance with the current task goals. It comprises a set of components including task switching, updating and response inhibition and coordinating multiple simultaneous operations. The fronto parietal network has been suggested as a neural basis for cognitive control. Liver transplantation is one of the most dynamic fields in modern medicine and is a lifesaving option for patients with end stage liver disease. Since the first successful transplantation in 1967, liver transplantation has been performed in many centers worldwide saving thousands of liver failure patients. Aim of the Work: based on that, our study aimed to assess cognitive functions, depression, and anxiety in patients with end stage liver disease before and after liver transplantation, then compare between both. Patients and Methods: the study involved convenience sample of 44 recipients with ESLD prepared for liver transplant, 30 of which completed the study and 14 cases dropped out. Results: all data were recorded and statistical analysis was done using the Statistical Package for Social Science SPSS20th version. The results were tabulated, grouped and statistically analyzed using the suitable statistical parameters. For description analysis. Conclusion: more liver impairment as estimated by Child Pugh score and MELD score associated with more impairment in cognitive tasks. In the current study the presence or absence of hepatic encephalopathy had no relation to cognitive function.
\end{abstract}

Keywords: Cognitive Functions; Executive Functions; Liver Transplant; Working Memory; Fronto Parietal Network.

\section{INTRODUCTION}

The liver is a vital organ that detoxifies most endogenous and exogenous toxic compounds, thus when the liver fails these processes do not proceed properly and toxic compounds may reach the brain and affect cerebral function ${ }^{(\mathbf{1}}$. Prolonged ingestion of alcohol as well as presence of chronic viral toxins like hepatitis B and C, both usually cause severe complications and lead to hyperdynamic system circulation as well as neuropsychiatric dysfunction and the severity of cognitive impairment is linked to the degree of hepatic dysfunction ${ }^{(2)}$. The CNS is usually involved during the development of chronic liver disease depending on its degree of severity. This involvement can cause limited mental capacity, changes in psychomotility and/or hepatic coma associated with irreversible brain damage ${ }^{(2)}$. Clinical presentation and pathophysiologic mechanisms of brain injury are dependent on the type of liver failure (fulminant or chronic). Recently evidence that hepatitis $\mathrm{C}$ virus can be found in the central nervous system and may have a role in a subcortical pattern of neuropsychological impairment in patients similar to that seen in HIV/AIDS dementia ${ }^{(3)}$. HE is frequently associated with a wide range of neuropsychiatric abnormalities and has been classified from minimal
HE (MHE) to different grades of overt HE, it is believed that cerebral edema is the common pathogenic mechanism for cognitive impairment in $\mathrm{MHE}$ and overt $\mathrm{HE}^{(4)}$. Patients with clinical HE show various neuropsychiatric symptoms including impairment in the sleep wake cycle, cognitive and intellectual function and motor activity and coordination as well as alteration in personality and consciousness. MHE may present by mild cognitive impairment, attention deficits, psychomotor slowing and impaired visuo-motor and bimanual coordination that can progress to clinical $\mathrm{HE}$ and reduces quality of life ${ }^{(1)}$. It is unclear whether minimal HE is fully reversible or persists to some degree after LT. Some prospective studies have shown that brain abnormalities detected in MRI normalize within one year after $\mathrm{LT}^{(5)}$. Liver transplantation (LT) can correct liver function resulting in an improvement in symptoms of MHE, however some cognitive deficits seem to persist to some degree. The MHE patients can experience persistent cognitive deficits after LT, in addition permanent brain injury has been found in those with previous episode of overt $\mathrm{HE}{ }^{(4)}$. HE occurring before LT can have a substantial negative impact on post-transplant outcomes, and preoperative history of HE may be a predictor of posttransplant neurologic complications. Even with resolution of 
previous episodes of overt or MHE some patients continue to experience cognitive deficits after transplant ${ }^{(6)}$.

\section{AIM OF THE WORK}

To assess cognitive functions in patients with end stage liver disease before liver transplantation. Assess cognitive functions of patients with end stage liver disease 3 months after liver transplantation. Also, to make Comparison between cognitive functions before and after liver transplantation.

\section{PATIENTS AND METHODS}

Subjects: 1) Study design: This is a prospective comparative study, 2) Study site: Subjects were recruited from liver transplant unit in the following centers: Ain Shams Center for Organ Transplant (ASCOT) in Ain Shams specialized hospital, 3ry center: A tertiary referral hospital is a hospital that provides tertiary care, which is health care from specialists in a large hospital to select cases for transplant from primary and secondary care hospitals; ASCOT selects about 30 patients/year for transplant with average 3-5 drop out. The location of applying the study was at the hospital itself at inpatients wards of liver transplantation unit, the instructor was sitting in front of the patient who is lying on his bed with a sliding table in front of him, some patient were able to move to the resident office which is present at the same floor few steps from the patient's room, 3) Study population: This study involved recipients prepared for liver transplant (ESLD) in liver transplant unit in the above mentioned center, according to the following criteria, after being tested through the preparatory phase in which the main instructor practiced the applied tools and insured that it's totally comprehensive and will not make patients with such general medical condition more exhausted, Inclusion criteria: The study included subjects with the following criteria: Patients with ESLD who are candidate for transplantation. Patients between 18 to 60 years of age. Patients of both genders male \& female. Patients who meet the above criteria signed an informed consent to participate in the research, Exclusion criteria: Patients currently having Hepatic Encephalopathy. Patients with major central neurological disease. Patients with unclear sensorium or delirious. Patients with psychotic disorder or mental disability. Patients with severe renal disease (Creatinine clearance $\leq 30 \mathrm{ml} / \mathrm{hr}$ ). Patients with severe pulmonary disease The refusal of patients to participate in the study. Child Pugh score class A, a) Sample design: We selected a Convenience sample, b) Sample size: Forty four patients with ESLD were selected. Sample size was calculated using EpiInfo ${ }^{\circledR}$ version 6.0 , setting the type- 1 error $(\alpha)$ at 0.05 and the power $(1-\beta)$ at 0.80 . 30 patients completed the study with 14 drop out cases. Data Management and Analysis: The collected data were revised, coded, tabulated and introduced to a PC using Statistical package for Social Science (SPSS 20). Data were presented and suitable analysis was done according to the type of data obtained for each parameter. The study was approved by the Ethics Board of Ain Shams University and an informed written consent was taken from each participant in the study. Descriptive statistics: Mean, Standard deviation $( \pm \mathrm{SD})$ and range for parametric numerical data, while Median and Interquartile range (IQR) for non parametric numerical data. Frequency and percentage of non-numerical data.

\section{RESULTS}

Table (1): Demographic data of the sample cases

\begin{tabular}{|c|c|c|c|}
\hline & & Mean & Standard Deviation \\
\hline \multicolumn{2}{|l|}{ Age } & 47.6 & 11.0 \\
\hline \multirow{2}{*}{ Marrital Status } & Married & 27 & $90.0 \%$ \\
\hline & Single & 3 & $10.0 \%$ \\
\hline \multirow{5}{*}{ Education } & Illiterate & 5 & $16.7 \%$ \\
\hline & Primary & 3 & $10.0 \%$ \\
\hline & Prep & 3 & $10.0 \%$ \\
\hline & Secondary & 2 & $6.7 \%$ \\
\hline & University & 17 & $56.7 \%$ \\
\hline \multirow{4}{*}{ Work } & No & 7 & $23.3 \%$ \\
\hline & Student & 1 & $3.3 \%$ \\
\hline & Semi Skilled & 8 & $26.7 \%$ \\
\hline & Skilled & 14 & $46.7 \%$ \\
\hline \multirow{2}{*}{ Residence } & Rural & 10 & $33.3 \%$ \\
\hline & Urban & 20 & $66.7 \%$ \\
\hline \multirow{2}{*}{ Gender } & Female & 13 & $43.3 \%$ \\
\hline & male & 17 & $56.7 \%$ \\
\hline \multirow{4}{*}{ Past M.H } & No & 17 & $56.7 \%$ \\
\hline & $\mathrm{DM}$ & 11 & $36.7 \%$ \\
\hline & Renal & 1 & $3.3 \%$ \\
\hline & Chest & 1 & $3.3 \%$ \\
\hline \multirow{2}{*}{$\mathrm{HCV}$} & Negative & 25 & $83.3 \%$ \\
\hline & Positive & 5 & $16.7 \%$ \\
\hline \multirow{2}{*}{ Past H.E } & Yes & 8 & $26.7 \%$ \\
\hline & No & 22 & $73.3 \%$ \\
\hline
\end{tabular}

Past M.H: past medical history, HCV: hepatitis C virus, Past H.E: past hepatic encephalopathy 
Table (2): Comparison between cases and drop out regarding demographic data

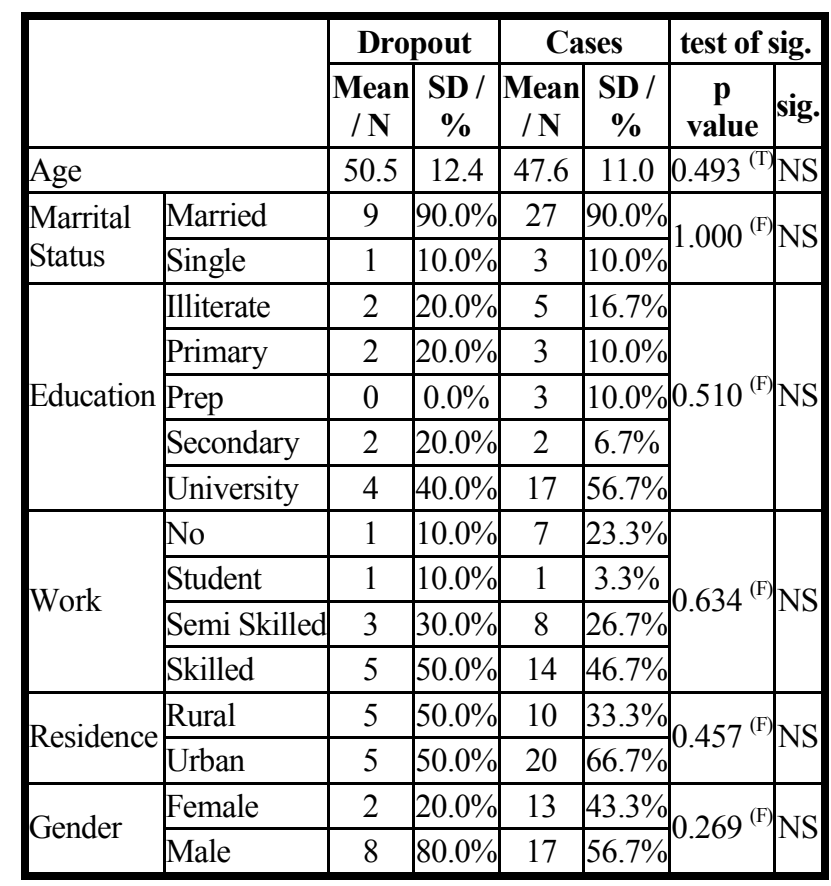

Table (3): Comparison between cases and drop out regarding Medical history

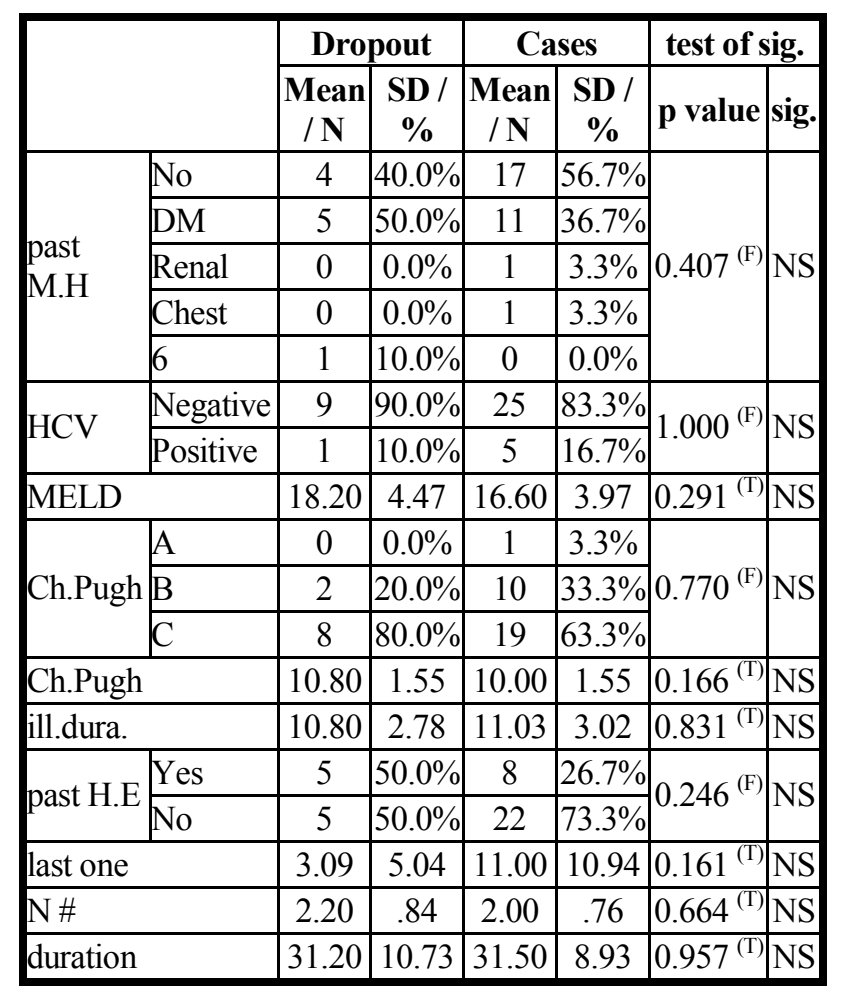

Marital S: marital status, Past M.H: past medical history, HCV: hepatitis C virus, Past H.E: past hepatic encephalopathy, Ch.Pugh: Child Pugh score, Ill.dura: illness duration of liver disease, N\#: number of episodes of hepatic encephalopathy, $\mathrm{T}$ : $\mathrm{t}$ test, F: Fisher exact test
Table (4): Quantitative Comparison between cases before and after liver transplantation.

\begin{tabular}{|c|c|c|c|c|c|c|c|c|}
\hline & & & \multicolumn{2}{|c|}{ Before } & \multicolumn{2}{|c|}{ After } & \multicolumn{2}{|c|}{$\begin{array}{c}\text { Paired } t \\
\text { test }\end{array}$} \\
\hline & & & Mean & SD & Mean & SD & $\begin{array}{c}\mathbf{p} \\
\text { value }\end{array}$ & sig. \\
\hline \multirow{2}{*}{ Intelligence } & \multirow{2}{*}{\multicolumn{2}{|c|}{\begin{tabular}{|l|} 
pict.compl \\
similarity \\
\end{tabular}}} & 10.1 & 2.8 & 10.9 & 2.7 & 0.125 & $\mathrm{NS}$ \\
\hline & & & 9.1 & 3.2 & 9.4 & 3.5 & 0.482 & $\mathrm{NS}$ \\
\hline \multirow{2}{*}{$\begin{array}{l}\text { trail making } \\
\text { test }\end{array}$} & \multicolumn{2}{|c|}{$\mathrm{B}$} & $210^{\mathrm{a}}$ & $\begin{array}{l}162- \\
290^{\mathrm{b}}\end{array}$ & $167^{\mathrm{a}}$ & $\begin{array}{l}108- \\
250^{\mathrm{b}}\end{array}$ & $\begin{array}{c}0.008 \\
\mathrm{M}\end{array}$ & $\mathrm{S}$ \\
\hline & \multicolumn{2}{|l|}{ A } & $85^{\mathrm{a}}$ & $\begin{array}{c}60- \\
120^{\mathrm{b}} \\
\end{array}$ & $65.5^{\mathrm{a}}$ & $\begin{array}{l}47- \\
91^{\mathrm{b}} \\
\end{array}$ & $\begin{array}{c}0.006 \\
\mathrm{M}\end{array}$ & $\mathrm{S}$ \\
\hline \multirow{5}{*}{$\begin{array}{l}\text { Wacselar } \\
\text { memory } \\
\text { scale }\end{array}$} & \multicolumn{2}{|c|}{ inform. } & 13.4 & 0.8 & 13.9 & 0.3 & 0.005 & $\mathrm{~S}$ \\
\hline & \multicolumn{2}{|c|}{ verb.PA1 } & 15.1 & 4.2 & 14.7 & 5.0 & 0.679 & $\mathrm{NS}$ \\
\hline & \multicolumn{2}{|c|}{ Verb. PA2 } & 5.9 & 1.5 & 6.0 & 1.7 & 0.682 & NS \\
\hline & \multirow{2}{*}{\begin{tabular}{|l|} 
Digit \\
Span
\end{tabular}} & forw & 7.7 & 2.7 & 10.4 & 13.5 & 0.286 & $\mathrm{NS}$ \\
\hline & & backw & 4.5 & 2.4 & 4.6 & 2.4 & 0.823 & $\mathrm{NS}$ \\
\hline \multirow{6}{*}{$\begin{array}{l}\text { Beneton } \\
\text { visual } \\
\text { retention test }\end{array}$} & \multicolumn{2}{|c|}{ OCS } & 3.2 & 2.4 & 4.0 & 3.0 & 0.083 & $\mathrm{NS}$ \\
\hline & \multicolumn{2}{|l|}{ ECS } & 7.1 & 1.4 & 7.1 & 1.4 & 0.326 & $\mathrm{NS}$ \\
\hline & \multicolumn{2}{|l|}{\begin{tabular}{|l} 
DIFF \\
\end{tabular}} & 3.9 & 2.2 & 3.5 & 2.0 & 0.337 & $\mathrm{NS}$ \\
\hline & \multicolumn{2}{|l|}{ OES } & 10.7 & 4.9 & 9.6 & 5.5 & 0.121 & NS \\
\hline & \multicolumn{2}{|l|}{ EES } & 3.8 & 2.0 & 4.0 & 2.1 & 0.246 & NS \\
\hline & \multicolumn{2}{|l|}{ DIFF } & 7.0 & 4.1 & 5.8 & 4.3 & 0.084 & $\mathrm{NS}$ \\
\hline \multicolumn{3}{|l|}{ HAD } & 13.4 & 5.6 & 11.0 & 6.9 & 0.074 & $\mathrm{NS}$ \\
\hline
\end{tabular}

a: Median, b: IQR (Inter quartile range), M: Wilcoxon signed Rank test, Pict. compl: picture completion, Inform: information, Verb.PA1: verbal association part 1, Verb.PA2: verbal association part 2, OCS: obtained corrected score, ECS: expected corrected score, Diff C: difference in corrected score, OES: obtained error score, EES: expected error score, Diff E: difference in error score, DS forward: digit span forward, DS backward : digit span backward

There was a significant improvement regarding trail making test part $\mathrm{A}$ and $\mathrm{B}$ and information of the Wacselar Memory Scale.

\section{DISCUSSION}

Postoperative cognitive dysfunction (POCD) in liver transplant (LT) recipients is defined as a "more than expected" postoperative deterioration in cognitive domains, including shortterm and long-term memory, mood, consciousness and circadian rhythm. It is diagnosed, after exclusion of other neurological complications, by using specific neuropsychological tests that need preoperative baseline ${ }^{(7)}$. Some advanced cirrhotic patients can develop hepatic encephalopathy (HE), which manifests as central nervous system abnormalities that can affect mental and motor function ${ }^{(8)}$. Primary aim of the current study was to compare cognitive function of patients undergoing liver transplantation (ESLD), before and after the operation. Administration of the following cognitive tasks was done 3-5 days before transplantation then repeated 3 months after 
transplantation.; the Wechsler Memory Scale, Benton Visual Retention Test and Trail Making Test (TMT) Parts A \& B were used to accomplish this task. The sample consisted of 30 cases \& 14 drop out. Our study included 30 cases, 17 of which were male and 13 females, the mean age of recipients was $47.6,90 \%$ of the sample were married and $10 \%$ are single. Concerning education, $16.7 \%$ can't read or write, where $56.7 \%$ have completed their university studies, $46,7 \%$ were skilled worker and most of patients lives in urban areas. Comparison between Pre \& Post transplant quantitative results: a) Sociodemographic data: Consistent with the current study Niazi et al., $2017^{\left({ }^{(9)}\right.}$ found no significant correlation between percent of change of TMT-A $(p=0.825) \& B(p=0.672)$ and information of WMS $(p=0.959)$ with age of cases. Furthermore they reported no correlation between age and cognitive performance at pre or post-transplant assessments, as young versus older recipients did not differ in cognitive rates of change for either memory or executive functioning. b) Comparison between cognitive tasks before and after transplantation: The Trail Making Test, parts A and B (TMT-A, B) was used to assess the rates of visual-motor processing and mental flexibility. In the current study there were statistically significant differences between the cases before and after liver transplantation regarding trail making test part $\mathrm{A}$ $(\mathrm{P}=0.008)$ and part $\mathrm{B}(\mathrm{P}=0.006)$, this conclusion agree with that found by Pegum et al., 2011 ${ }^{(10)}$ who showed Significant improvements on TMT versions $\mathrm{A}$ and $\mathrm{B}$ (assessing visual tracking, visuomotor speed, and visual alternating attention) at follow-up assessment with $\mathrm{P} \leq 0.002$ for both TMT-A \& B and a trend was found toward improvement in overall immediate recall (General Memory of WMS revised), ( $\mathrm{P} \leq 0.057)$. Regarding information collected in our study using Wecselar memory scale in our study it showed statistically significant results between cases before and after liver transplantation $(\mathrm{P}=0.005$. This result concur that observed by Pegum et al., 2011 ${ }^{(10)}$ in which a trend was found toward improvement in overall immediate recall (General Memory of WMS revised), ( $\mathrm{P} \leq 0.057)$. In addition Pegum et al., 2011 (10) showed significant improvement on the Visual Memory Index of WMS-R similar to what found in the presented study which showed no statistically significant results between pre and post- liver transplant, this inconsistency may be due to selection of patients in the current study where most of patients were $\mathrm{HCV}$ positive and this may affect cognitive function and may be irreversible more than alcohol related liver disease. c) Relation to stage of liver disease: In the current study the mean of child Pugh score in drop out patients were $(\mathrm{A}=0, \mathrm{~B}=2, \mathrm{C}=8)$ and that of cases were $(A=1, B=10, C=19)$ with no significant difference between them regarding Digits Forward (DF) \&Digits Backward (DB). Furthermore, there was no significant correlation between trail making test part A \& B and information of the WMS and Child Pugh score. Contrary to PANTIGA et al., $2003^{(11)}$, who found significant differences between the four experimental groups A, B, C, OLT (Child Pugh A$\mathrm{B}-\mathrm{C}$-orthotopic liver transplant) and the control group (GC) in both digit span forward \& backward $(\mathrm{P}=0.000)$, significant differences were also found between the transplant recipients and the cirrhotic patients with Child-Pugh stage $\mathrm{C}$ regarding the Digit span backward test (C vs. OLT), On the Digits Forward (DF) and Digits Backward (DB) tests, subjects with OLT achieved higher direct scores than the groups with cirrhosis, this inconsistency maybe due to different groups of comparison. d) Hospital anxiety \& depression scale: The Hospital Anxiety and Depression scale HADS was used in our study to assess patients having symptoms of depression and/or anxiety as this may be a confounding factor that might negatively affect recipients 'cognitive functions. Our results showed no significant improvement regarding the Hospital Anxiety and Depression scale HADS $(\mathrm{p}=0.074)$, that was in contrast to Ishihara et al., $2013^{(12)}$, where the Hospital Anxiety and Depression scale HADS scores improved significantly among the 12 patients after liver transplantation, this different results may be due to lower number of patients and longer period of follow up (6 months) after liver transplant in the other study.

\section{CONCLUSION}

Although there was more impairment regarding cognitive function of dropout patients $(25 \%)$ but that was not statistically significant. High percentage of dropout among the current study indicating the importance of reaching a protocol for psychiatric and cognitive assessment prior to liver transplantation as that may help to 
determine who will get benefit more from surgery regarding this dimension.

\section{CONFLICTS OF INTEREST}

There are no conflicts of interest.

\section{REFERENCES}

1. Felipo V (2013): Hepatic encephalopathy: effects of liver failure on brain function. Nature Reviews Neuroscience,14: 851-858.

2. Brodersen C, Koen E, Ponte A et al. (2014): Cognitive function in patients with alcoholic and nonalcoholic chronic liver disease. The Journal of Neuropsychiatry and Clinical Neurosciences, 26:241-248.

3. Sorrel J, Zolnikov B, Sharma A et al. (2006): Cognitive impairment in people diagnosed with end-stage liver disease evaluated for liver transplantation. Journal of psychiatry and clinical neurosciences, 60:174-181.

4. Lin W, Chou K, Chen $C$ et al. (2014): Longitudinal brain whie matter alterations in minimal hepatic encephalopathy before and after liver transplantation. Journal PLoS ONE, 9: 1-9.

5. Gracia-Martinez R, Rovira A, Alonso J et al. (2011): Hepatic encephalopathy is associated with posttransplant cognitive function and brain volume. Liver transplantation, 17: 38-46.

6. Teperman LW (2013): Impact of pretransplant hepaticencephalopathy on liver posttransplantation outcomes. International Journal of Hepatology, 10: 1-9.
7. Aceto P, Perilli V, Lai C et al. (2014): Postoperative cognitive dysfunction after liver transplantation, Gen Hosp Psychiatry, 37(2):109-115.

8. Cheng Y, Huang $L$, Zhang $X$ et al. (2015): Liver transplantation nearly normalizes brain spontaneous activity and cognitive function at 1 month: a resting-state functional MRI study. Metabolic brain disease, 30: 979-988.

9. Niazi S, Ferman T, Keaveny A et al. (2017): Impact of age on memory and executive function in liver transplant recipients. Journal of psychomotor research., 97:163.

10. Pegum N, Connor J, Feeny $G$ et al. (2011): Neuropsychological functioning in patients with alcohol-related liver disease before and after liver transplantation. Transplantation, 92:13711377.

11. Pantiga $C$, Rodrigo $L$, Cuesta $M$ et al. (2003): Cognitive deficits in patients with hepatic cirrhosis and in liver transplant recipients. The journal of neuropsychiatry and clinical neuroscience, 15:84-89.

12. Ishihara T, Ito M, Niimi Y et al. (2013): Clinical and radiological impact of liver transplantation for brain in cirrhosis patients without hepatic encephalopathy. Clinical neurology and neurosurgery, 115: 2341-2347. 